\title{
Do mail-shots improve access to primary care for young men with depression?
}

\author{
Paul Walters, MRC Fellow \\ James Fisher, Research Associate \\ André Tylee, Professor \\ Section of Primary Care Mental Health, \\ Health Services Research Department, \\ Institute of Psychiatry, \\ King's College London, \\ London, SE5 8AF \\ UNITED KINGDOM
}

\begin{abstract}
Background: Young men with mental health problems often do not present to traditional primary care services. Novel methods to engage this population need to be explored. Between April and July 2005, Croydon Primary Care Trust developed a depression service for men aged 18 to 35 years. Part of this service was a mail-shot to all men in this age group registered with two general practices. The mail-shot informed them of the symptoms of depression, the importance of seeking help and where to obtain help. The objective of this research was to determine whether this mail-shot influenced the number of young men presenting to primary care with depression.

Method: This was a before and after study. The quarterly incidences of depression in men aged 18 to 35 years was calculated from January 2000 to June 2005. Incidence risk ratios were calculated to compare pre-mail-shot incidence with the post mail-shot incidence. The cost of the mail-shot was calculated.

Results: There were 148 new cases of depression diagnosed between January 2000 and June 2005 across the practices. There was a statistically significant increase in depressed young men contacting the two participating general practices in the quarter after the mailshot compared with previous quarters (IRR 2.57; 95\% CI 1.59-4.17; p < 0.001). The cost of the mail-shot was $£ 1.00$ per registered man aged between 18 and 35 years, or $£ 297$ $(450 €)$ per case of depression detected.

Conclusion: Mail-shots may be a cost-effective way to encourage this traditionally 'hard-to-reach' group to consult primary care professionals for depression treatment.
\end{abstract}




\section{Background and Objectives}

In the United Kingdom, the majority of common mental illness is treated in primary care $^{1}$. Young men have the lowest consultation rates in primary care ${ }^{2}$ and as suicide is one of the commonest causes of death in young men their tendency to consult infrequently may be a contributing factor ${ }^{3}$. Nearly five thousand people kill themselves annually, of whom $80 \%$ are men, $75 \%$ of these are aged between 15 and 24 years ${ }^{4}$. Depression is a major risk factor for suicide ${ }^{5,6}$, and evidence suggests that the risk of suicide when depressed is greater in those who are not in contact with health services ${ }^{7}$. It therefore seems important to improve access to primary care for young men with depression.

Access to primary care services can be understood in terms of the pathways to care model of Goldberg and Huxley ${ }^{8}$. This model consists of filters through which patients must pass in order to receive the required treatment. The first filter is the decision to attend primary care and is determined by the patient's help seeking behaviour and the accessibility of primary care professionals.

A number of recent primary care initiatives in the UK have sought to facilitate the patient's passage through this first filter by introducing novel services and personnel. These have included Walk-in Centres, and new primary care personnel such as the Graduate Primary Care Mental Health Workers (GPCMHW). The GPCMHWs have had a variety of roles including compiling diverse local health service knowledge for sign-posting within health services, starting proactive mental health awareness campaigns, and providing brief therapeutic interventions 9 .

The reasons why young men consult primary care less are complex ${ }^{10}$. There are pragmatic and service issues that may limit accessibility to care. Young men in employment may find it particularly difficult to attend primary care and may not be familiar with a practice or the practitioners. Walk-In Centres in contrast may offer more flexibility in terms of opening times and anonymity ${ }^{11}$. Salisbury et al. compared attendance between GP surgeries and Walk-In Centres, and concluded that they improve access to care, but not necessarily for those with greatest health needs ${ }^{11}$. Walk-in users were more likely to be white and of higher education, than GP attendees. It is unlikely that these centres will improve access for the male population most at risk of suicide.

An alternative may be to improve access to traditional primary care services by advertising and highlighting depression and the help available. Symonds et al. developed a practice nurse-led depression service ${ }^{12}$. All patients registered with the practice were informed about this new service by a mail-shot. The mail-shot consisted of a flyer documenting the symptoms of depression, and information on how to contact the service. They found an increase in the number of people who contacted the service in direct response to the flyer suggesting that raising awareness of a new service by sending a flyer may increase attendance ${ }^{12}$.

In April 2005, Croydon Primary Care Trust (PCT) and South London \& Maudsley NHS Trust established a novel depression service aimed at improving access and enhancing depression care for young men aged 18 to 35 years. It was run by two GPCMHWs in two large South London Health Centres (the Brigstock Medical Practice and the Norbury Health Centre) with a total combined practice population of 19200 patients. Both health centres serve relatively deprived populations (Index of Multiple deprivation scores 22 and 20 respec- 
tively). As part of this service all men aged between 18 and 35 years registered with the practice were sent information about depression and the service in the form of a mail-shot. They were invited to contact the GPCMHW if appropriate.

The objective of this study was to evaluate whether a mail-shot about depression increased the number of young men accessing primary care for depression treatment.

\section{Methods}

This was a before and after study design. In April 2005, a letter and a depression booklet were delivered to every man aged $18-35$ years registered with the Brigstock Medical Practice and the Norbury Health Centre. The letter provided information about depression and contact details for the GPCMHW. The depression booklet contained further information about the symptoms of depression and the importance of seeking help. Men contacting the GPCMHW, were managed according to a depression treatment protocol developed by Croydon PCT (details available from authors).

The incidence of young men presenting with new depression to both practices as ascertained from computer records was calculated for each quarter from January 2000 to June 2005. The mail-shot was sent in April 2005. The number of presenting men in the quarter April-June, 2005 (post-mail-shot) was then compared with previous quarters to determine the effect of the intervention (the mail-shot). Considering the number of men presenting with depression in every quarter over five years, means that any artefactual change in quarter to quarter fluctuations could be controlled for. These were calculated from the practices electronic medical records.
The total number of men aged between 18 and 35 years registered with the practice in each quarter from January 2000 to June 2005 was also calculated from these records.

Incident risk ratios (IRR) for presenting with depression in the quarter after the mailshot compared with the preceding quarters were calculated by Poisson regression using Stata 8.0 (Statacorp, Texas). IRRs were also calculated for each second quarter compared to each first quarter annually. This was to determine whether any effect could have been due to a confounding factor such as seasonal variation between adjacent quarters. The direct cost of the mail-shot was calculated.

Ethical approval was obtained from London and Surrey Borders Local Research Ethics Committee.

\section{Results}

Between April and June, 2005, 2672 men aged between 18 and 35 years were registered with the two practices. From the $1^{\text {st }}$ January 2000 to the $30^{\text {th }}$ June 2005, 148 men (aged 18-35 years) presented with new cases of depression to the two practices as recorded by their GP. Ninety-nine $(66.9 \%)$ attended at least one follow-up appointment in the two months following presentation (mean number of follow-ups 2.27; s.d = 1.87). The mean age of the men was 27.6 years $(s . d=5.16)$.

The number of incident cases per quarter is shown in Table I. Nineteen men contacted the two surgeries in the 3 months following the mail-shot (April-June, 2005). Nine contacted the depression service directly and of these, 3 (33\%) were referred by the GPCMHW to the GP following the depression protocol. A further 10 men with depression contacted 
the GP directly in response to the mail-shot rather than the GPCMHW.

The IRR of presenting with depression in the quarter after the mail-shot intervention (April-July 2005) compared with all quarters prior to the mail-shot intervention was 2.57 ([95\% CI 1.59-4.17]; $\mathrm{p}<0.001$ ). There was no statistically significant difference in the IRR of presenting with depression in any of the quarters (2000-2005) prior to the mail-shot (IRR 0.98; [95\% CI 0.96-1.01]; $\mathrm{p}=0.234$ ). The IRR of presenting with depression in the quarter after the mail-shot intervention compared with the quarter immediately prior to the mail-shot (January to April, 2005) was 6.25 ([95\% CI 1.85-21.13]; $\mathrm{p}=0.003$ ).
The IRRs for the $1^{\text {st }}$ and $2^{\text {nd }}$ quarters in consecutive years are shown in Table II. There were no significant differences in IRRs between the first and second quarters in any of the years prior to 2005 (intervention year).

Each mail shot cost approximately $£ 1.00$ to produce and send. A total of 2672 were sent across both centres. Given that 19 young men presented to primary care with depression in the quarter after the mail shot, each new presentation costs about $£ 140$. Of the 19 men, 11 contacted the depression service. If only these are included in the economic analysis the cost of the mail shot alone is about $£ 297$ per new presentation to the depression service.

Table I

Incidence of GP recording depression in young men (aged 18-35 years) per 1000 registered young men per year.

\begin{tabular}{|c|c|c|c|c|}
\hline Year & Quarter & $\begin{array}{c}\text { Incident } \\
\text { cases }\end{array}$ & $\begin{array}{l}\text { Total male* } \\
\text { practice } \\
\text { population }\end{array}$ & $\begin{array}{c}\text { Incidence/1000 } \\
\text { practice population } \\
\text { men*/year }\end{array}$ \\
\hline \multirow[t]{4}{*}{2000} & 1 & 1 & 2058 & \multirow[t]{4}{*}{4.8} \\
\hline & 2 & 2 & 2071 & \\
\hline & 3 & 2 & 2080 & \\
\hline & 4 & 5 & 2089 & \\
\hline \multirow[t]{4}{*}{2001} & 1 & 9 & 2103 & \multirow[t]{4}{*}{13.6} \\
\hline & 2 & 8 & 2119 & \\
\hline & 3 & 5 & 2120 & \\
\hline & 4 & 7 & 2129 & \\
\hline \multirow[t]{4}{*}{2002} & 1 & 3 & 2139 & \multirow[t]{4}{*}{9.8} \\
\hline & 2 & 7 & 2167 & \\
\hline & 3 & 6 & 2207 & \\
\hline & 4 & 6 & 2243 & \\
\hline \multirow[t]{4}{*}{2003} & 1 & 9 & 2285 & \multirow[t]{4}{*}{13.0} \\
\hline & 2 & 12 & 2322 & \\
\hline & 3 & 6 & 2409 & \\
\hline & 4 & 5 & 2454 & \\
\hline \multirow[t]{4}{*}{2004} & 1 & 12 & 2482 & \multirow[t]{4}{*}{12.5} \\
\hline & 2 & 7 & 2521 & \\
\hline & 3 & 9 & 2617 & \\
\hline & 4 & 5 & 2649 & \\
\hline \multirow[t]{2}{*}{2005} & 1 & 3 & 2711 & \multirow[t]{2}{*}{$6.9 * *$} \\
\hline & 2 & 19 & 2746 & \\
\hline
\end{tabular}

\footnotetext{
* Age 18-35 years.
}

** 6 month incidence. 
Table II

IRRs of first versus second quarters in successive years.

\begin{tabular}{cccc} 
Year & IRR for $2^{\text {nd }}$ Quarter vs $1^{\text {st }}$ Quarter & $95 \%$ CI & p-value \\
\hline 2000 & 2.00 & {$[0.18-21.74]$} & 0.575 \\
2001 & 0.89 & {$[0.34-2.29]$} & 0.796 \\
2002 & 2.30 & {$[0.60-8.91]$} & 0.227 \\
2003 & 1.31 & {$[0.36-1.54]$} & 0.429 \\
2004 & 0.58 & {$[0.23-1.45]$} & 0.244 \\
2005 & 6.3 & {$[1.85-21.13]$} & 0.003
\end{tabular}

Overall IRR (second quarter 2005, compared to prior quarters) 2.57 ([95\% CI 1.59-4.17]; $\mathrm{p}<0.001$ ).

\section{Conclusion}

This evaluation has a number of limitations that need to be taken into consideration. The number of young men who contacted the service was small which has implications for the precision of the IRRs. The mail-shot and service was only conducted at two centres limiting generalisabilty. An increase in the IRRs in the quarter following the mail-shot compared to quarter immediately before the mail-shot could be due to three factors. It could be due to a decrease in the incidence of depression in the $1^{\text {st }}$ quarter, with no increase in the incidence in the quarter following the mail shot; it could be due to a smaller decrease in incidence in the 1st quarter and a small but nonsignificant increase in the post-mail shot quarter; or it could be due to a significant increase in incidence following the mail shot. In this evaluation there was statistically significant increase in incidence after the mail shot in the second quarter of 2005 compared to the first quarter of 2005 which may in part be due to a smaller incidence in the quarter before the mail shot.

It is not possible from this pilot to say with certainty that the increase in young men presenting with depression was due to the mail-shot or the depression service, and it is quite possible that confounding variables, such as time of year, depression awareness campaigns, media articles or other factors that may have affected presentation may have confounded the results. However, we were not aware of any such campaigns in the quarter immediately after the mail-shot. We have also tried to control, as far as possible, for these factors by measuring the incidence of depression in young men presenting to the two centres in the preceding 5 years. Considering that more men contacted their GP rather than the new service directly after receiving the mail-shot, it is likely that this is an effect of the mail-shot. Outcome data was not collected in this evaluation so it is not possible to determine whether the service had a beneficial effect for the young men who used it.

Despite these limitations, this study provides evidence that a mail-shot and depression service set up specifically for depressed 18-35 year old men, increases the incidence of young men with depression presenting to primary care. These results support a more general finding by Symons et al., that depressed patients do respond to mailshots $^{12}$. Targeted mail-shots may therefore be an effective tool to improve access for depression care services.

Mail-shots may be effective for a number of reasons. They may directly influence the decision to attend primary care, or they may help 
men recognise the symptoms of depression and thus prompt them to consult. Recognising these symptoms as part of a depressive illness may be the first step in the help-seeking decision making process. Re-framing the seemingly disparate symptoms as a depressive illness may give them 'permission' to them to seek help. It is interesting that over the 5 years, nearly a third of young men diagnosed with depression failed to return for a follow-up appointment with their GP. A longer follow-up may also have identified young men who acted on the information in the mail-shot later, perhaps when it became more appropriate.

The mail shots were printed, prepared and distributed at a cost of $£ 1$ per patient. However, this did not include the costs of administering a service for patients that consequentially present with depression and a full costing of this work would have to include staff costs, along with the costs of maintaining contact with young men.

The implication of this study is that novel methods of widening access to primary care merit further research. This is necessary to identify the important parts of the service and to determine the effectiveness of the individual components of the service (i.e. is the mail shot effective without the depression service, and vice versa). This study did not determine the outcomes of men who contacted the GPCMHW service compared to those who contacted their GP directly, or the reasons why the young men consulted the GPCMHW or the GP.

\section{Acknowledgement}

We would like to acknowledge the help of the staff and patients at the Brigstock Medical Practice and the Norbury Health Centre.
In particular, we would like to acknowledge the help of John Haseler, Kate Mages, Sarah Kildea, Dipti Ghandi, Simon Vearnals, Dr M. Whitehead and Dr N. Chaudury.

\section{Reference}

1. Goldberg D \& Huxley P Common mental disorders. A biosocial model. London: Routledge; 1992.

2. McCormick A, Fleming D, and Charlton J. Morbidity Statistics in General Practice: Fourth National Study 19911992. MB5 No.3. London: HMSO; 1995.

3. Brock A \& Rooney C. Trends in the mortality of young adults aged 15-44 in England and Wales, 1961 to 2001. Health Stat Q 2003; 19: 22-54.

4. Department of Health. National Suicide Prevention Strategy for England. London: Department of Health; 2002.

5. Hawton K. Sex and suicide. Gender differences in suicidal behaviour. Br J Psychiatry 2000; 177: 484-485.

6. Foster T, Gillespie K, McClelland R. Mental disorders and suicide in Northern Ireland. Br J Psychiatry 1997; 170: 447-452.

7. Rihmer Z. Strategies of suicide prevention: focus on health care. J Affect Disord 1996; 39(2): 83-91.

8. Goldberg D, Huxley P. Mental illness in the community. the pathway to psychiatric care. London: Tavistock; 1980.

9. Bower P, Jerrim S, Gask L. Primary care mental health workers: role expectations, conflict and ambiguity. Health Soc Care Community 2004; 12(4): 336-345.

10. Walinder J, Rutz W. Male depression and suicide. Int Clin Psychopharmacol 2001; 16: s21-s24.

11. Salisbury C, Munro J. Walk-in centres in primary care: a review of the international literature. Br J Gen Pract 2003; 53(486): 53-59.

12. Symons L, Tylee A, Mann A, Jones R, Plummer S, Walker $\mathrm{M}$ et al. Improving access to depression care: descriptive report of a multidisciplinary primary care pilot service. Br J Gen Pract 2004; 54(506): 679-683.

Address for correspondence:

P Walters

p.walters@iop.kcl.ac.uk 